\title{
Genomic Regions Involved in Differences in Eating and Cooking Quality Other than $W x$ and Alk Genes between indica and japonica Rice Cultivars
}

Kiyosumi Hori $^{1 *+} \mathbb{D}$, Keitaro Suzuki ${ }^{1+}$, Haruka Ishikawa ${ }^{2}$, Yasunori Nonoue ${ }^{1}$, Kazufumi Nagata ${ }^{1,3}$, Shuichi Fukuoka ${ }^{1}$ and Junichi Tanaka ${ }^{1,4^{*}+}$

\begin{abstract}
Background: In temperate rice cultivation regions, japonica rice cultivars are grown preferentially because consumers deem them to have good eating quality, whereas indica rice cultivars have high grain yields and strong heat tolerance but are considered to have poor eating quality. To mitigate the effects of global warming on rice production, it is important to develop novel rice cultivars with both desirable eating quality and resilience to high temperatures. Eating quality and agronomic traits were evaluated in a reciprocal set of chromosome segment substitution lines derived from crosses between a japonica rice cultivar 'Koshihikari' and an indica rice cultivar 'Takanari'.

Results: We detected 112 QTLs for amylose and protein contents, whiteness, stickiness, hardness and eating quality of cooked rice grains. Almost of 'Koshihikari' chromosome segments consistently improved eating quality. Among detected QTLs, six QTLs on chromosomes 1-5 and 11 were detected that increased whiteness and stickiness of cooked grains or decreased their hardness for 3 years. The QTLs on chromosomes 2-4 were not associated with differences in amylose or protein contents. QTLs on chromosomes 1-5 did not coincide with QTLs for agronomic traits such as heading date, culm length, panicle length, spikelet fertility and grain yield. Genetic effects of the detected QTLs were confirmed in substitution lines carrying chromosome segments from five other indica cultivars in the 'Koshihikari' genetic background.

Conclusion: The detected QTLs were associated with differences in eating quality between indica and japonica rice cultivars. These QTLs appear to be widely distributed among indica cultivars and to be novel genetic factors for eating quality traits because their chromosome regions differed from those of the GBSSI (WX) and SSIla (Alk) genes. The detected QTLS would be very useful for improvement of eating quality of indica rice cultivars in breeding programs.
\end{abstract}

Keywords: Rice, Eating quality, Quantitative trait loci, Chromosome segment substitution lines

\footnotetext{
*Correspondence: horikiyo@affrc.go.jp; tanajun@affrc.go.jp

${ }^{\dagger}$ Kiyosumi Hori, Keitaro Suzuki and Junichi Tanaka contributed equally to this

work.

'Institute of Crop Science, NARO, 2-1-2 Kannondai, Tsukuba, Ibaraki 305-8518,

Japan

Full list of author information is available at the end of the article
}

\section{Springer Open}

(c) The Author(s). 2021 Open Access This article is licensed under a Creative Commons Attribution 4.0 International License, which permits use, sharing, adaptation, distribution and reproduction in any medium or format, as long as you give appropriate credit to the original author(s) and the source, provide a link to the Creative Commons licence, and indicate if changes were made. The images or other third party material in this article are included in the article's Creative Commons licence, unless indicated otherwise in a credit line to the material. If material is not included in the article's Creative Commons licence and your intended use is not permitted by statutory regulation or exceeds the permitted use, you will need to obtain permission directly from the copyright holder. To view a copy of this licence, visit http://creativecommons.org/licenses/by/4.0/. 


\section{Background}

Rice (Oryza sativa L.) is a staple food for nearly half of the world population (GriSP (Global Rice Science Partnership), 2013). This species is divided into two main subspecies, indica and japonica, which differ in their morphological and physiological characteristics (Khush 1997). Indica is grown mainly in tropical regions, whereas japonica is grown in temperate regions such as China, Korea and Japan. The two subspecies have been suggested to have different domestication origins because, for example, seed shattering is reduced by the loss of function of different genes (Konishi et al. 2006; Li et al. 2006) and there are multiple sequence polymorphisms throughout their genomes (Garris et al. 2005; Wang et al. 2018), indicating considerable intraspecific differentiation. Generally, $F_{1}$ plants from crosses between the two subspecies show low fertility, and segregating populations such as $\mathrm{F}_{2}$ often show sterility and hybrid weakness (Matsubara et al. 2007; Yamamoto et al. 2007). Therefore, in modern rice breeding, programs for indica-background breeding and for japonicabackground breeding are separate, although breeders use common agronomically important genes for disease resistance, plant height and stress tolerance in these programs.

The International Rice Research Institute (IRRI) developed an indica cultivar 'IR8' by introducing the semidwarf gene $s d 1$ derived from 'Dee-geo-woo-gen'; 'IR8' has played the main role in the Green Revolution in rice (Khush 1999). Since then, IRRI has developed many high-yielding indica cultivars (e.g., 'IR64'), which are widely cultivated, mainly in tropical regions (Mackill and Khush 2018). Japanese indica cultivars such as 'Takanari' and 'Hokuriku 193' are derived from crosses with the IRRI cultivars and have significantly higher yields than Japanese japonica cultivars such as 'Koshihikari', even in temperate climates (Imbe et al. 2004; Goto et al. 2009). Global warming is likely to reach $1.5^{\circ} \mathrm{C}$ between 2030 and 2052 if it continues at the current rate (IPCC (Intergovernmental Panel on Climate Change), 2018). In general, indica cultivars are adapted to higher temperatures in low latitudes better than japonica cultivars. Therefore, the genetic backgrounds of indica rice will be more suitable for cultivation in temperate regions, which are likely to experience an increase in temperature during rice growth seasons in the future (IPCC (Intergovernmental Panel on Climate Change), 2018).

Indica rice cultivars are preferred by consumers in most rice cultivation areas, but not in Northeast Asia, where people are accustomed to eating japonica rice (Juliano et al. 1993; Calingacion et al. 2014). Many consumers in such countries as China, Korea and Japan tend to prefer the softness and strong stickiness of cooked grains of japonica rice cultivars. Almost all indica cultivars have low stickiness and high hardness of cooked grains (Calingacion et al. 2014; Hori and Yano 2013). One of main genetic factors controlling stickiness and hardness of cooked rice grains are allelic differences in the $\operatorname{Waxy}(W x, G B S S I)$ gene involved in amylose synthesis in rice endosperm (Tan et al. 1999; Wan et al. 2004; Tian et al. 2005; Takeuchi et al. 2007; Su et al. 2011; Yang et al. 2018; Park et al. 2019; Yang et al. 2020). In general, indica cultivars have the $W x^{a}$ allele, which results in high amylose content, whereas japonica cultivars have the $W x^{b}$ allele, which results in moderate amylose content (Juliano et al. 1993). Several other Wx alleles have also been reported in indica cultivars such as $W x^{l \nu}$ and $W x^{i n}$ with high amylose content, and in japonica cultivars such as $W x^{l a}$ and $W x^{m q}$ with low amylose content (Sato et al. 2002; Zhou et al. 2015; Zhang et al. 2019; Zhou et al. 2020). Chromosome region at the $W x$ gene showed high recombination rate and various gene alleles were generated by intragenic recombination. The Alkali degradation (Alk, SSIIa) gene involved in amylopectin chain elongation is also controlling eating quality of cooked rice grains by altering starch characteristics such as amylose content, gelatinization temperature and gel consistency (Umemoto et al. 2002; Umemoto 2018). Haplotype analysis revealed that these phenotypic differences were significantly correlated with allelic differences of the Alk gene between indica and japonica rice cultivars (Umemoto et al. 2004). In general, indica cultivars have the Alk allele, which is strong functional allele and results in high gelatinization temperature, whereas japonica cultivars have the alk allele, which is weak functional allele and results in low gelatinization temperature. Another $A l k$ allele of $A l k^{b}$ is reported as weak functional allele with low gelatinization temperature and gel consistency both in indica and japonica cultivars (Chen et al. 2020). Allelic difference of the Alk gene largely changed on eating and cooking qualities in near-isogenic lines (NILs) introducing the $W x^{b}$ and $w x$ gene alleles as compared with in NILs introducing the $W x^{a}$ gene allele (Umemoto 2018).

Amylose and protein contents of a Japanese indica cultivar 'Takanari' are not much different from those of the japonica cultivar 'Koshihikari', which is a leading cultivar in Japan (Hori et al. 2016; Iijima et al. 2019). However, 'Takanari' has the $W x^{b}$ allele introduced from japonica cultivars (Aoki et al. 2015). 'Takanari' has harder cooked grains and significantly inferior taste in comparison with typical Japanese japonica rice cultivars including 'Koshihikari'. Therefore, the difference in eating quality between indica and japonica rice cultivars cannot be explained only by amylose and protein contents, but other major genetic factors related to eating quality are hardly known. To improve eating quality of indica rice cultivars, it is necessary to detect novel genetic factors associated with eating quality. 
indica rice cultivars are considered unsuitable for consumers in Northeast Asia because of their eating and cooking characteristics. Development of novel rice cultivars with the indica genetic background, good eating quality and high yield in Northeast Asia would be an effective solution to the possible food-supply crisis caused by global warming in the future. In this study, we attempted to detect QTLs for eating quality in chromosome segment substitution lines (CSSLs) derived from crosses between 'Koshihikari' and 'Takanari' (Takai et al. 2014). We found multiple QTLs related to differences in eating quality between the two cultivars, and some of these QTLs were not associated with amylose or protein contents. These CSSLs can be promising materials to introduce novel genetic factors for eating quality into indica rice cultivars.

\section{Materials and Methods \\ Plant Materials}

To detect QTLs involved in the control of eating-quality traits, we used a reciprocal set of CSSLs derived from crosses between a japonica rice cultivar 'Koshihikari' and an indica rice cultivar 'Takanari' (Takai et al. 2014). Forty-one CSSLs covered most of the 'Takanari' genome in the 'Koshihikari' genetic background and 39 CSSLs covered the 'Koshihikari' genome in the 'Takanari' genetic background. Genotype information of individual CSSLs is available in Takai et al. (2014).

Eight CSSLs derived from crosses between 'Koshihikari' as a recurrent parent and five indica rice cultivars-'Naba' (WRC5), 'Bleiyo' (WRC63), 'Bei Khe' (WRC3), 'Tupa 1213' (WRC32) and 'Basilanon' (WRC44) (Kojima et al. 2005) - were selected to investigate whether the detected QTLs for eating quality traits were common among indica rice cultivars. In these CSSLs of the 'Koshihikari' genetic background, chromosome segments derived from these indica rice cultivars cover the regions of detected QTLs. Genotype information of the eight CSSLs were indicated in Supplementary Table S1.

To reveal the genotypes of the QTL ( $q W H 1$ ) region that enhances the whiteness of cooked rice grains, which is adjacent to the $s d 1$ gene, we selected five leading cultivars in Japan: 'Koshihikari', 'Hitomebore', 'Hinohikari', 'Akitakomachi' and 'Nanatsuboshi' (Kobayashi et al. 2018), and six high-yielding and good-eating-quality cultivars that were released recently (2011-2019) in Japan: 'Akidawara', 'Hoshijirushi', 'Mizuhonokagayaki', 'Tsukiakari', 'Natsuhonoka' and 'Nijinokirameki'.

\section{Evaluation of Eating Quality Traits}

All plants of CSSLs and parental cultivars 'Koshihikari' and 'Takanari' were grown in an experimental field at the Institute of Crop Science, NARO, Tsukubamirai, Japan $\left(36.01^{\circ} \mathrm{N}, 140.02^{\circ} \mathrm{E}\right)$. CSSLs of the 'Koshihikari' genetic background were grown in 2016 and 2017, and CSSLs of the 'Takanari' genetic background in 2018. Japanese leading cultivars and recently developed cultivars were grown in 2018. One-month-old seedlings of all CSSLs and cultivars were transplanted in mid-May at one plant per hill in plots with a double row for each line; there was $15 \mathrm{~cm}$ between plants and $30 \mathrm{~cm}$ between rows. Cultivation management followed the standard procedures used at the institute.

Eating quality traits were evaluated by instrumental methods according to Hori et al. (2016) and Iijima et al. (2019). Apparent amylose content was determined by using an Auto Analyzer II (Bran+Luebbe Co. Ltd., Norderstedt, Germany). Crude protein content was determined by the combustion method with an induction furnace at $900{ }^{\circ} \mathrm{C}$ (American Association of Cereal Chemists International, Approved Method 46-30.01). Whiteness and grain qualities were evaluated with a Rice Grain Analyzer RGQI20B (Satake Co., Ltd., Hiroshima, Japan). Eating quality score was measured in a Cooked Rice Taste Analyzer STA1A (Satake Co., Ltd.). This instrument has been used to estimate eating quality scores by measuring transmitted light volume and reflection light volume of cooked rice grains under three wavelengths (Mikami 2009). Physical properties of cooked grains were measured by the high-compression/lowcompression method with a Tensipresser MyBoy texture analyzer (Takemoto Electric Co., Tokyo, Japan). These instrumental methods showed significant correlations with the eating quality scores by the sensory tests (Okadome 2005; Mikami 2009; Kwon et al. 2011; Hori et al. 2016).

\section{Scoring of Agronomic Traits}

For each CSSL and parental cultivar, days to heading was defined as the number of days from sowing to heading of half of the plants. Culm length, panicle length, panicle number, spikelet fertility (ratio of the number of sterile and fertile grains per panicle) and unhulled grain weight per plant were measured for five plants per CSSL and parental cultivar at maturity stage.

\section{Statistical and Genetic Analyses}

Eating quality traits and agronomic traits of CSSLs were compared with those of each recurrent parent, 'Koshihikari' or 'Takanari', by using the Dunnett's multiple comparison procedure provided by the JMP 11 software (SAS Institute Inc., NC, USA). In the Dunnett's tests, 'Koshihikari' was used as a control for 41 CSSLs of 'Takanari' and 8 CSSLs of other indica cultivars in the 'Koshihikari' genetic background, and 'Takanari' was used as a control for 39 CSSLs in the 'Takanari' genetic background. QTLs were declared present when 
individual trait scores were significantly different between the line and the recurrent parent.

\section{DNA Marker Genotyping}

Total genomic DNA of Japanese leading rice cultivars and recently developed rice cultivars was extracted from leaves using the CTAB method (Hori et al. 2012) and a DNA sui-sui $\mathrm{S}$ kit (Rizo Inc., Tsukuba, Ibaraki, Japan). The DNA markers described in Bao et al. (2006) and Hiratsuka et al. (2010) were used for determining the Alk gene allele in 'Koshihikari' and 'Takanari'. We selected eight DNA markers-simple sequence repeats, insertion/deletions (InDels) and the $s d 1$ gene-that were polymorphic between 'Koshihikari' and 'Takanari'. Simple sequence repeat markers of RM11716, RM11837, RM12168 and RM12263 were selected from IRGSP (International Rice Genome Sequencing Project) (2005). Oligoribonucleotide sequences of InDel markers and the $s d 1$ gene marker were 5'-GTGATCAATGTCGAGATA ACGTTCC-3' and 5'-GGTAAAAGGATTAGAGCA CCGCTAC-3' (JI_indel_01), 5'-TTTCAGGTAGGCAT CACCAATAGAG-3' and 5'-CTCCGTCCGAGGTGTC ATAAATTAG-3' (JI_indel_02), 5'-ATGCCGTTAA TAGAATGGGAATACG-3' and 5'-AGATCAAATC GTCAATGTGGAACAC-3' (JI_indel_03), and 5' -ACGC ACGGGTTCTTCCAGGTGT- ${ }^{\prime}$ ' and $5^{\prime}$-GAGCGG GAGGCGGAAGAAGTC-3' $(s d 1)$.

\section{Results}

QTLs for Eating Quality Traits in CSSLs of the 'Koshihikari' Genetic Background

The phenotypes of the CSSLs of the 'Koshihikari' genetic background varied widely in eating quality traits (amylose and protein contents, eating quality score, stickiness and hardness of cooked rice grains, and grain whiteness; Table 1, Supplementary Tables S2 and S3, Supplementary Figure 1). In comparison with 'Koshihikari', 44 QTLs in 2016 and 32 QTLs in 2017 were associated with significant differences in all six eating quality traits analyzed (Table 2). Genomic regions of almost all QTLs were consistent between 2016 and 2017.

We focused on six of the detected QTLs (Fig. 1): $q W H 1$ for grain whiteness on the long arm of chromosome 1, qST2 for stickiness of cooked grains on the long arm of chromosome 2, qST3-1 for stickiness of cooked grains on the short arm of chromosome 3, qHA4-2 for hardness of cooked grains on the long arm of chromosome 4, qST5 for stickiness of cooked grains on the long arm of chromosome 5 and qHA11 for hardness of cooked grains on the long arm of chromosome 11. We selected these six QTLs because of their large genetic effects and detection in both years.

In comparison with 'Koshihikari', SL1204, carrying $q W H 1$, had significantly higher whiteness, amylose content and hardness of cooked grains, but lower stickiness of cooked grains in 2016 and 2017. SL1208, carrying $q S T 2$, had significantly lower eating quality score, stickiness of cooked grains and grain whiteness. SL1210, carrying $q S T 3-1$, had significantly lower eating quality score and stickiness of cooked grains. SL1217, carrying $q H A 4-2$, had significantly lower eating quality score and higher hardness of cooked grains and grain whiteness. SL1219, carrying qST5, had significantly lower eating quality score and stickiness of cooked grains but higher amylose content and grain whiteness. SL1237, carrying qHA11, had significantly lower eating quality score, stickiness of cooked grains and amylose content, hardness of cooked grains and grain whiteness. The 'Takanari' allele of $q W H 1$ increased grain whiteness. The 'Koshihikari' alleles of the remaining five QTLs resulted in high eating quality score and in strong stickiness and softness of cooked grains. qST2, qST3-1 and qHA4-2 were not associated with differences in amylose or protein contents in both years.

Eating quality traits are easily affected by many agronomic traits including heading date, grain size and weight, grain number per panicle and spikelet fertility (Juliano et al. 1993; Iijima et al. 2019). We evaluated agronomic traits of the CSSLs, in particular those of the six CSSLs each carrying a QTL on chromosomes 1-5 and 11. In comparison with 'Koshihikari', five lines showed no significant differences in days to heading (flowering time), culm length, panicle length, number of panicles, unhulled grain weight or spikelet fertility (Supplementary Tables S2 and S3). However, one line, SL1208, showed weak vigor, including few panicles and low unhulled grain weight and spikelet fertility.

\section{QTLs for Eating Quality Traits in CSSLs of the 'Takanari' Genetic Background}

Among the CSSLs of the 'Takanari' genetic background, there was also a wide range of phenotypic differences in eating quality traits (Table 3, Supplementary Table S4, Supplementary Figure 1). In comparison with 'Takanari', 36 QTLs in 2018 showed significant differences in the six eating quality traits (Table 4). The six QTLs detected in the 'Koshihikari'-background CSSLs on chromosomes 1-5 and 11 coincided well with those detected in the 'Takanari' CSSLs (Fig. 1).

In comparison with 'Takanari', SL1303, carrying $q W H 1$, had significantly higher grain whiteness. Thus, this chromosome region increased whiteness both in the 'Koshihikari' and 'Takanari' genetic backgrounds. SL1306, carrying qST2, had significantly higher eating quality score, stickiness of cooked grains and grain whiteness. SL1308, carrying qST3-1, had significantly higher eating quality score and grain stickiness. SL1315, carrying qHA4-2, had significantly higher eating quality 


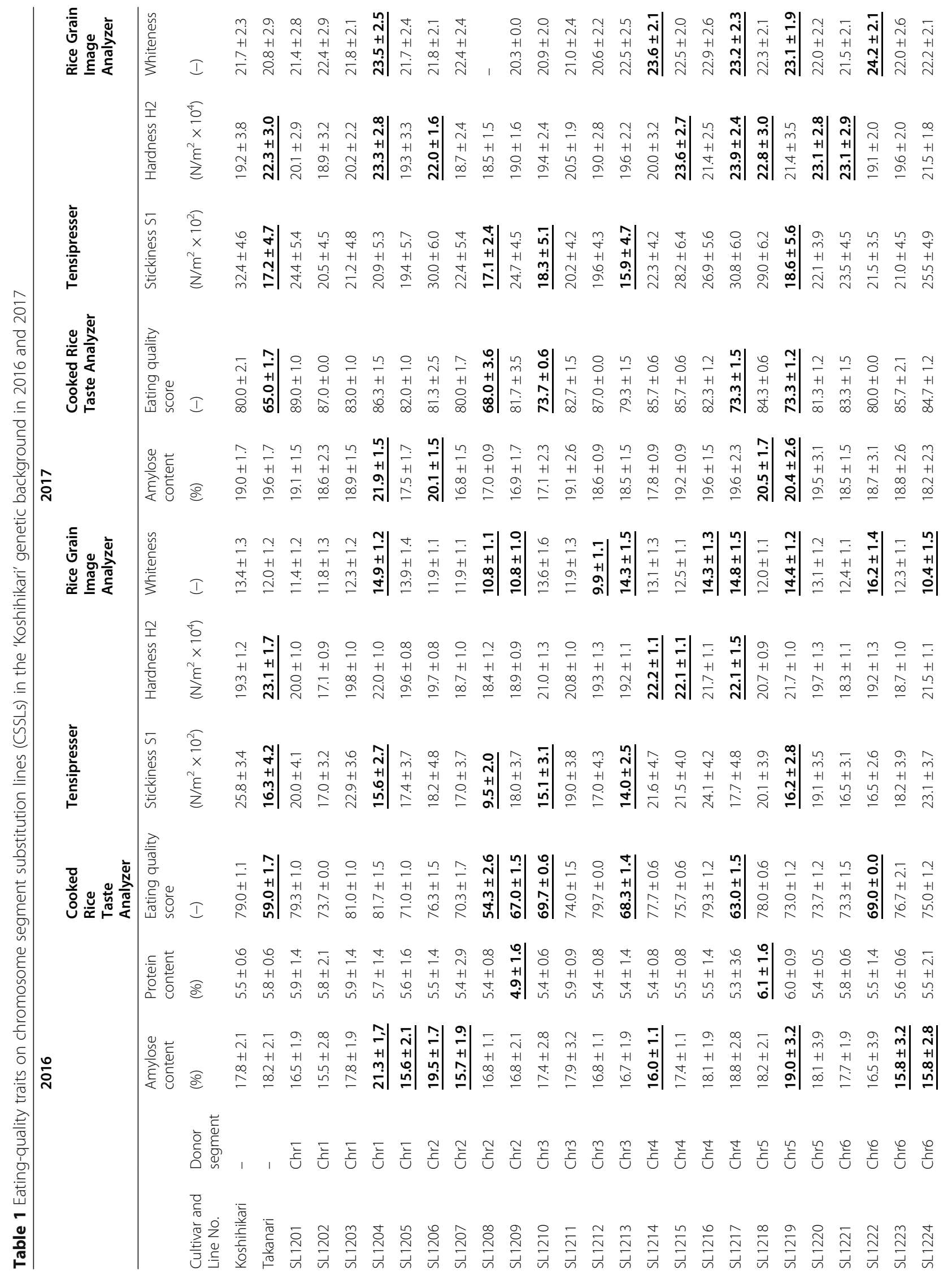




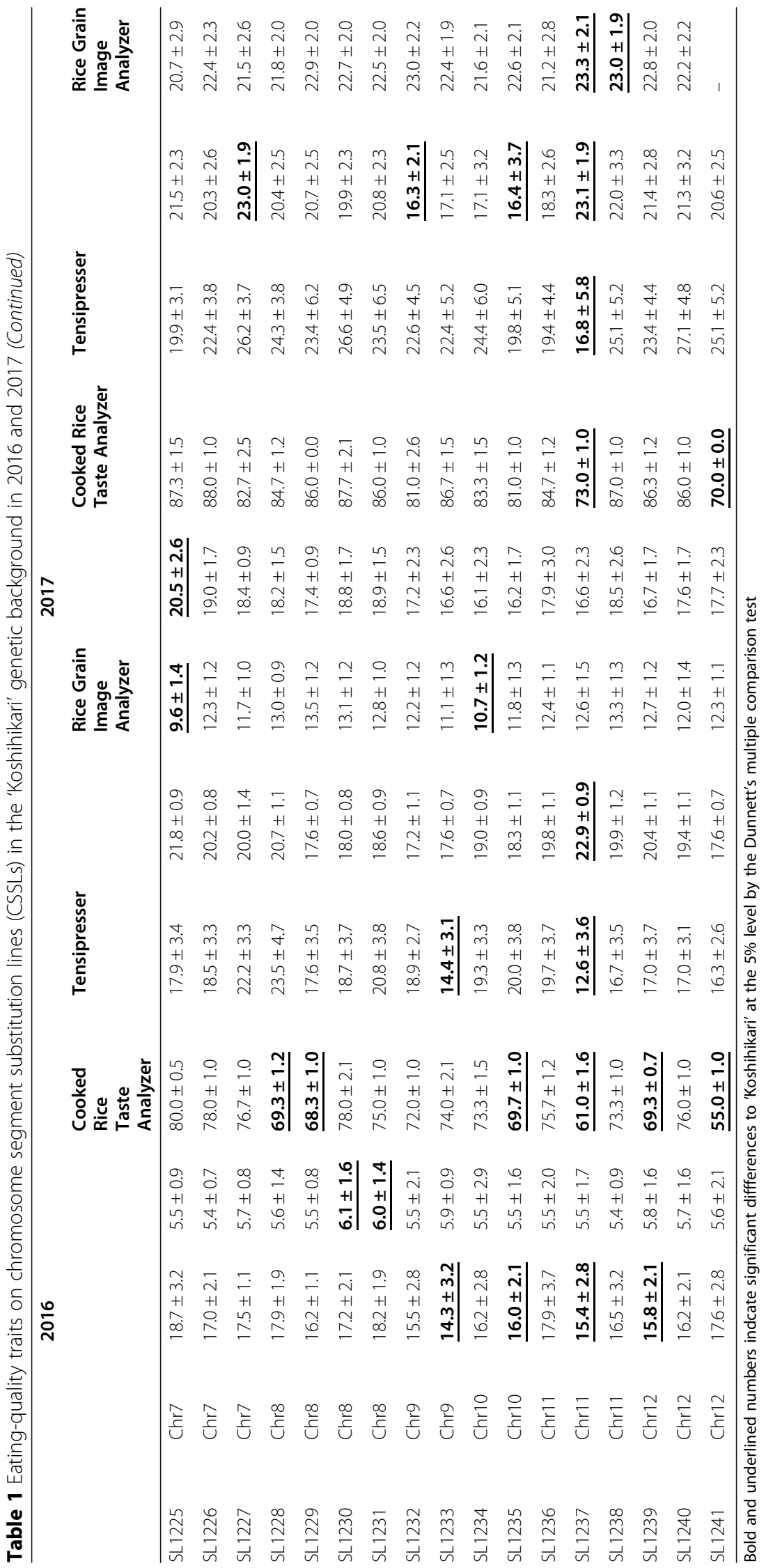


Table 2 QTLs for eating-quality traits on CSSLs in the 'Koshihikari' genetic background in 2016 and 2017

\begin{tabular}{|c|c|c|c|c|c|c|}
\hline Trait & Locus name & CSSLS & Position & Flanking marker interval & Year & Additive effect \\
\hline \multirow[t]{14}{*}{ Amylose content (\%) } & $q A C 1-1$ & SL1204 & Chr1 & RM1196-RM7594 & 2016 & 1.8 \\
\hline & & & & & 2017 & 1.5 \\
\hline & $q A C 1-2$ & SL1205 & & RM6648-RM6321 & 2016 & -1.1 \\
\hline & $q A C 2-1$ & SL1206, SL1207 & Chr2 & RM5897-RM1234 & 2016 & 0.9 \\
\hline & & & & & 2017 & 0.6 \\
\hline & $q A C 4$ & SL1214 & Chr4 & RM16260-RM1305 & 2016 & -0.9 \\
\hline & $9 A C 5$ & SL1219 & Chr5 & RM6034-RM1386 & 2016 & 0.6 \\
\hline & & & & & 2017 & 0.8 \\
\hline & $q A C 6$ & SL1223, SL1224 & Chr6 & RM1340-RM1370 & 2016 & -1.0 \\
\hline & $q A C 7$ & SL1225 & Chr7 & RM4584-RM5481 & 2017 & 0.8 \\
\hline & qAC9 & SL1233 & Chr9 & RM6235-RM6797 & 2016 & -1.8 \\
\hline & $9 A C 10$ & SL1235 & Chr10 & RM4455-RM6673 & 2016 & -0.9 \\
\hline & $q A C 11$ & SL1237 & Chr11 & RM3701-RM1341 & 2016 & -1.2 \\
\hline & $9 A C 12$ & SL1239 & Chr12 & Bb77A02-RM2935 & 2016 & -1.0 \\
\hline \multirow[t]{3}{*}{ Protein content (\%) } & $q P C 2$ & SL1209 & Chr2 & RM6933-RM3850 & 2016 & -0.3 \\
\hline & $q P C 5$ & SL1218 & Chr5 & RM6034-RM1386 & 2016 & 0.3 \\
\hline & $q P C 8$ & SL1230, SL1231 & Chr8 & RM3634-RM4997 & 2016 & 0.3 \\
\hline \multirow[t]{16}{*}{ Eating quality score $(-)$} & $q E Q 2$ & SL1208, SL1209 & Chr2 & RM3515-RM3850 & 2016 & -12.4 \\
\hline & & & & & 2017 & -6.0 \\
\hline & qEQ3-1 & SL1210 & Chr3 & RM7332-RM5748 & 2016 & -4.7 \\
\hline & & & & & 2017 & -3.2 \\
\hline & qEQ3-2 & SL1213 & & RM2334-RM7389 & 2016 & -5.4 \\
\hline & qEQ4 & SL1217 & Chr4 & RM3839-RM5608 & 2016 & -8.0 \\
\hline & & & & & 2017 & -3.4 \\
\hline & qEQ5 & SL1219 & Chr5 & RM6034-RM1386 & 2017 & -3.4 \\
\hline & qEQ6 & SL1222 & Chr6 & RM5855-RM7193 & 2016 & -5.0 \\
\hline & qEQ8 & SL1228, SL1229 & Chr8 & RM6369-RM22709 & 2016 & -5.4 \\
\hline & qEQ10 & SL1235 & Chr10 & RM4455-RM6673 & 2016 & -4.7 \\
\hline & qEQ11 & SL1237 & Chr11 & RM3701-RM1341 & 2016 & -9.0 \\
\hline & & & & & 2017 & -3.5 \\
\hline & qEQ12-1 & SL1239 & Chr12 & Bb77A02-RM2935 & 2016 & -4.9 \\
\hline & $q E Q 12-2$ & SL1241 & & RM3326-RM1226 & 2016 & -12.0 \\
\hline & & & & & 2017 & -5.0 \\
\hline \multirow[t]{11}{*}{ Stickiness S1 $\left(\mathrm{N} / \mathrm{m}^{2} \times 10^{2}\right)$} & qST1 & SL1204 & Chr1 & RM1196-RM7594 & 2016 & -5.1 \\
\hline & qST2 & SL1208 & Chr2 & RM1211-RM3316 & 2016 & -8.2 \\
\hline & & & & & 2017 & -7.7 \\
\hline & qST3-1 & SL1210 & Chr3 & RM7332-RM5748 & 2016 & -5.4 \\
\hline & & & & & 2017 & -7.1 \\
\hline & qST3-2 & SL1213 & & RM2334-RM7389 & 2016 & -5.9 \\
\hline & & & & & 2017 & -8.3 \\
\hline & qST5 & SL1219 & Chr5 & RM6034-RM1386 & 2016 & -4.8 \\
\hline & & & & & 2017 & -6.9 \\
\hline & qST9 & SL1233 & Chr9 & RM6235-RM6797 & 2016 & -5.7 \\
\hline & qST11 & SL1237 & Chr11 & RM3701-RM1341 & 2016 & -6.6 \\
\hline
\end{tabular}


Table 2 QTLs for eating-quality traits on CSSLs in the 'Koshihikari' genetic background in 2016 and 2017 (Continued)

\begin{tabular}{|c|c|c|c|c|c|c|}
\hline Trait & Locus name & CSSLs & Position & Flanking marker interval & Year & Additive effect \\
\hline & & & & & 2017 & -7.8 \\
\hline \multirow[t]{14}{*}{ Hardness $\mathrm{H} 2\left(\mathrm{~N} / \mathrm{m}^{2} \times 10^{4}\right)$} & qHAl & SL1204 & Chr1 & RM1196-RM7594 & 2017 & 2.1 \\
\hline & qHA2 & SL1206 & Chr2 & RM6842-RM5699 & 2017 & 1.4 \\
\hline & qHA4-1 & SL1214, SL1215 & Chr4 & RM5414-RM5633 & 2016 & 1.5 \\
\hline & & & & & 2017 & 2.2 \\
\hline & qHA4-2 & SL1217 & & RM3839-RM5608 & 2016 & 1.4 \\
\hline & & & & & 2017 & 2.4 \\
\hline & qHA5-1 & SL1218 & Chr5 & RM1248-RM3838 & 2017 & 1.8 \\
\hline & qHA5-2 & SL1220 & & RM1386-RM3286 & 2017 & 2.0 \\
\hline & qHA6-1 & SL1221 & Chr6 & RM6467-RM5855 & 2017 & 2.0 \\
\hline & qHA7 & SL1227 & Chr7 & RM6394-RM7601 & 2017 & 1.9 \\
\hline & qHA9 & SL1232 & Chr9 & RM23654-RM6235 & 2017 & -1.5 \\
\hline & qHA1O & SL1235 & Chr10 & RM4455-RM6673 & 2017 & -1.4 \\
\hline & qHA11 & SL1237 & Chr11 & RM3701-RM1341 & 2016 & 1.8 \\
\hline & & & & & 2017 & 2.0 \\
\hline \multirow[t]{15}{*}{ Whiteness (-) } & qWH1 & SL1204 & Chr1 & RM1196-RM7594 & 2016 & 0.8 \\
\hline & & & & & 2017 & 0.9 \\
\hline & $q W H 2-1$ & SL1208, SL1209 & Chr2 & RM3515-RM3850 & 2016 & -1.3 \\
\hline & qWH3 & SL1212, SL1213 & Chr3 & RM3513-RM6970 & 2016 & 0.5 \\
\hline & qWH4-1 & SL1214 & Chr4 & RM16260-RM1305 & 2017 & 1.0 \\
\hline & qWH4-2 & SL1216, SL1217 & & RM1359-RM3916 & 2016 & 0.7 \\
\hline & & & & & 2017 & 0.8 \\
\hline & qWH5 & SL1219 & Chr5 & RM6034-RM1386 & 2016 & 0.5 \\
\hline & & & & & 2017 & 0.7 \\
\hline & qWH6-1 & SL1222 & Chr6 & RM5855-RM7193 & 2016 & 1.4 \\
\hline & & & & & 2017 & 1.3 \\
\hline & qWH6-2 & SL1224 & & RM5957-RM5463 & 2016 & -1.5 \\
\hline & qWH7 & SL1225 & Chr7 & RM4584-RM5481 & 2016 & -1.9 \\
\hline & qWH10 & SL1234 & Chr10 & RM7492-RM1859 & 2016 & -1.4 \\
\hline & qWH11 & SL1237, SL1238 & Chr11 & RM5824-RM6623 & 2017 & 0.8 \\
\hline
\end{tabular}

Positive additive effect means 'Takanari' allele increasing the trait values

score and lower hardness of cooked grains. SL1317, carrying $q S T 5$, had significantly higher eating quality score, stickiness of cooked grains and protein content, and lower hardness of cooked grains. SL1336, carrying qHA11, had significantly higher eating quality score, amylose content, protein content and grain stickiness, and lower hardness of cooked grains. The 'Koshihikari' alleles of all of the six QTLs resulted in high eating quality score, strong stickiness and softness of cooked grains, and high grain whiteness in the 'Takanari' genetic background. qST2, qST3-1 and $q H A 4-2$ were not associated with significant differences in amylose or protein contents.

We also evaluated agronomic traits in the CSSLs of the 'Takanari' genetic background. In comparison with
'Takanari', SL1303, SL1306, SL1308, SL1315 and SL1317 showed no significant differences in days to heading, culm length, panicle length, number of panicles or unhulled grain weight (Supplementary Table S4). However, SL1336 showed weak vigor including late flowering, short culms and panicles, few panicles and low unhulled grain weight.

\section{Confirmation of the Effects of QTLs in CSSLs of Other indica Cultivars}

To investigate whether the six detected QTLs would be commonly detected in segregating populations derived from crosses between japonica and other indica rice cultivars, we developed additional CSSLs carrying chromosome segments introduced from indica cultivars 'Naba', 


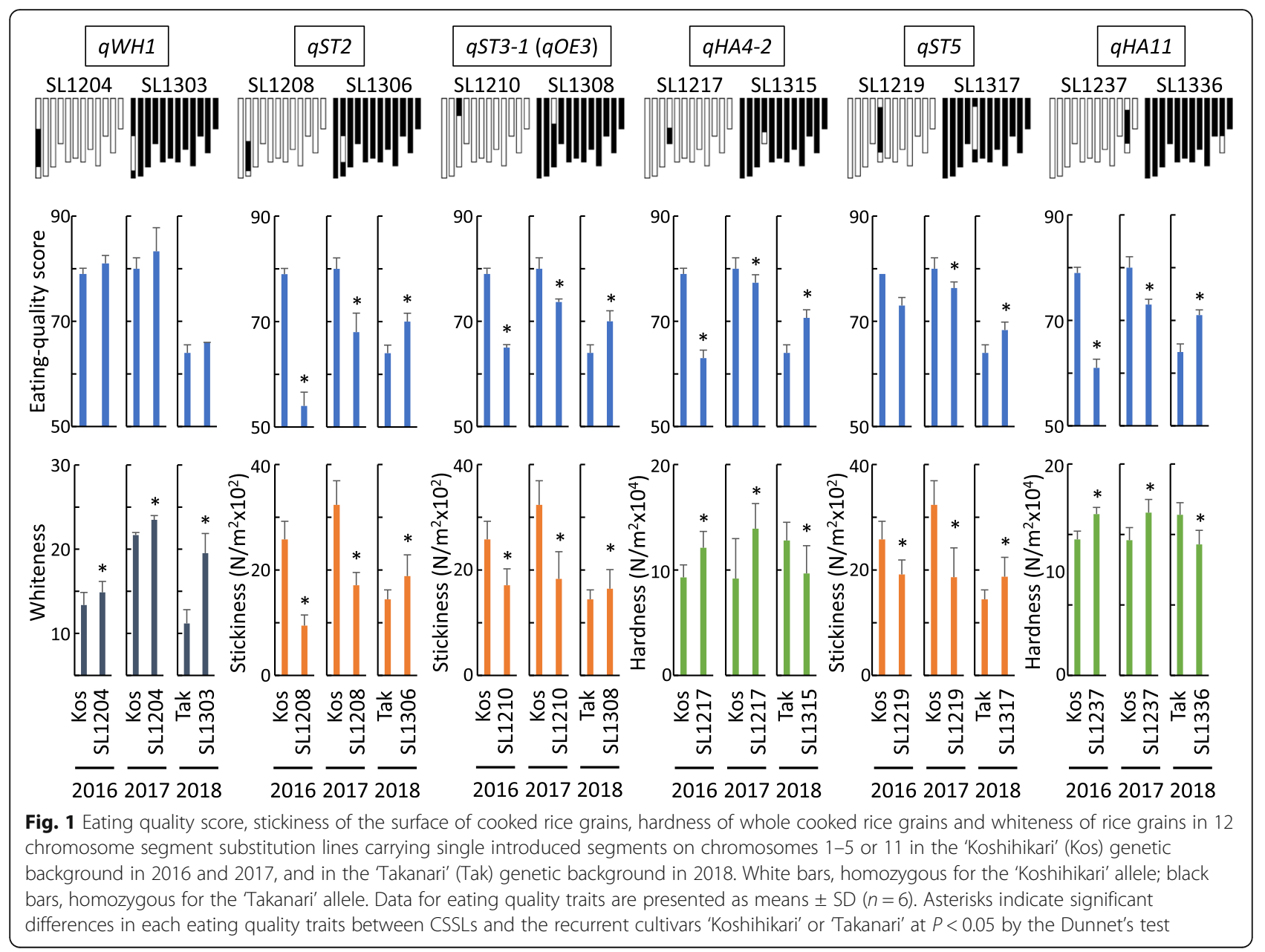

'Bleiyo', 'Bei Khe', 'Tupa 121-3' and 'Basilanon' in the 'Koshihikari' genetic background. Many of the eating quality traits of these CSSLs were significantly different from those of 'Koshihikari' (Fig. 2, Supplementary Table S5).

SL3202 and SL3302 each had a single segment on the long arm of chromosome 1 derived from 'Naba' and 'Bleiyo', respectively. In both lines, grain whiteness was significantly higher than in 'Koshihikari'. High whiteness in SL3202 and SL3302 was the same phenotype as that of SL1204 carrying $q W H 1$ from 'Takanari' (Fig. 1). Eating quality scores of SL3202 and SL3302 were not significantly different from that of 'Koshihikari'. In comparison with 'Koshihikari', hardness of cooked grains was higher in SL3202 and SL3302, as it was in SL1204 in 2017. SL3205 had a single segment on the long arm of chromosome 2 derived from 'Naba'. The eating quality score and stickiness of cooked grains were significantly lower in SL3205 than in 'Koshihikari'. These phenotypes were the same as that of SL1208 carrying qST2 from 'Takanari'. SL3315, SL2412 and SL2520 each had chromosome segments on the long arm of chromosome 4 derived from 'Bleiyo', 'Bei Khe' and 'Tupa 121-
3', respectively. SL3315 showed significantly lower eating quality score and higher hardness of cooked grains than those of 'Koshihikari'. SL2412 had a significantly lower eating quality score, but higher stickiness and hardness of cooked rice grains. SL2520 showed significantly higher grain whiteness and stickiness and hardness of cooked grains, but its eating quality score was not significantly different from that of 'Koshihikari'. The phenotypes of SL3315, SL2412 and SL2520 (low eating quality score and stickiness, and high hardness of cooked grains) were similar to those of SL1217, which had qHA4-2 from 'Takanari'. SL2544 and SL3042 each had a single segment on the long arm of chromosome 11 derived from 'Tupa 121-3' and 'Basilanon', respectively. SL2544 had lower eating quality score, amylose content and stickiness of cooked grains, and higher hardness of cooked grains. SL3042 had lower eating quality score and higher hardness of cooked grains. These phenotypes were similar to those of SL1237, which had qHA11 from 'Takanari'.

The QTLs in the CSSLs derived from crosses with other indica rice cultivars were detected in the same 
Table 3 Eating-quality traits on CSSLs in the 'Takanari' genetic background in 2018

\begin{tabular}{|c|c|c|c|c|c|c|c|}
\hline \multirow[b]{4}{*}{ Line No. } & \multirow[b]{4}{*}{ Donor segment } & \multicolumn{6}{|l|}{2018} \\
\hline & & \multirow{3}{*}{$\begin{array}{l}\text { Amylose } \\
\text { content } \\
(\%)\end{array}$} & \multirow{3}{*}{$\begin{array}{l}\text { Protein } \\
\text { content } \\
(\%)\end{array}$} & \multirow{3}{*}{$\begin{array}{l}\text { Cooked Rice } \\
\text { Taste Analyzer } \\
\text { Eating quality } \\
\text { score } \\
(-)\end{array}$} & \multirow{3}{*}{$\begin{array}{l}\text { Tensipresser } \\
\text { Stickiness S1 } \\
\left(\mathrm{N} / \mathrm{m}^{2} \times 10^{2}\right)\end{array}$} & \multirow{3}{*}{$\begin{array}{l}\text { Hardness } \mathrm{H} 2 \\
\left(\mathrm{~N} / \mathrm{m}^{2} \times 10^{4}\right)\end{array}$} & \multirow{3}{*}{$\begin{array}{l}\text { Rice Grain Image } \\
\text { Analyzer } \\
\text { Whiteness } \\
(-)\end{array}$} \\
\hline & & & & & & & \\
\hline & & & & & & & \\
\hline Takanari & - & $15.8 \pm 2.3$ & $5.7 \pm 1.1$ & $64.0 \pm 1.5$ & $14.4 \pm 4.0$ & $22.8 \pm 1.7$ & $11.2 \pm 1.6$ \\
\hline Koshihikari & - & $15.0 \pm 2.3$ & $5.1 \pm 1.1$ & $84.3 \pm 1.0$ & $20.5 \pm 3.1$ & $19.4 \pm 1.2$ & $14.7 \pm 2.6$ \\
\hline SL1301 & Chr1 & $17.0 \pm 2.0$ & $5.9 \pm 1.0$ & $67.3 \pm 1.2$ & $13.5 \pm 2.6$ & $20.9 \pm 1.6$ & $14.3 \pm 2.1$ \\
\hline SL1302 & Chr1 & $16.8 \pm 3.0$ & $5.5 \pm 1.5$ & $\underline{74.0 \pm 1.6}$ & $14.7 \pm 3.3$ & $23.0 \pm 1.3$ & $15.7 \pm 2.2$ \\
\hline SL1303 & Chr1 & $13.7 \pm 2.0$ & $5.9 \pm 1.0$ & $66.0 \pm 1.3$ & $12.8 \pm 2.7$ & $22.0 \pm 1.0$ & $\underline{19.5 \pm 2.3}$ \\
\hline SL1304 & Chr1 & $18.0 \pm 2.0$ & $5.5 \pm 1.0$ & $69.7 \pm 2.1$ & $14.6 \pm 1.2$ & $21.8 \pm 1.7$ & $14.5 \pm 2.5$ \\
\hline SL1305 & Chr2 & $14.5 \pm 2.3$ & $5.5 \pm 1.1$ & $67.0 \pm 1.7$ & $14.3 \pm 2.5$ & $20.2 \pm 1.1$ & $14.3 \pm 2.7$ \\
\hline SL1306 & Chr2 & $16.5 \pm 2.0$ & $5.5 \pm 1.0$ & $70.0 \pm 1.6$ & $16.8 \pm 2.4$ & $19.0 \pm 1.2$ & $16.3 \pm 2.1$ \\
\hline SL1307 & Chr2 & $16.7 \pm 2.0$ & $5.4 \pm 1.0$ & $69.0 \pm 1.7$ & $12.0 \pm 3.4$ & $21.7 \pm 1.4$ & $13.6 \pm 2.6$ \\
\hline SL1308 & Chr3 & $16.8 \pm 1.2$ & $5.5 \pm 0.6$ & $70.1 \pm 2.0$ & $16.6 \pm 3.6$ & $20.2 \pm 1.6$ & $15.0 \pm 2.4$ \\
\hline SL1309 & Chr3 & $16.4 \pm 2.3$ & $5.2 \pm 1.1$ & $66.3 \pm 1.5$ & $15.5 \pm 3.8$ & $22.6 \pm 1.5$ & $15.5 \pm 2.3$ \\
\hline SL1310 & Chr3 & $14.3 \pm 2.0$ & $5.4 \pm 1.5$ & $67.7 \pm 1.5$ & $16.5 \pm 3.3$ & $20.5 \pm 1.4$ & $13.5 \pm 2.9$ \\
\hline SL1311 & Chr3 & $19.2 \pm 2.5$ & $5.9 \pm 1.7$ & $68.7 \pm 1.2$ & $16.5 \pm 4.6$ & $23.3 \pm 1.6$ & $16.8 \pm 2.7$ \\
\hline SL1312 & Chr4 & $16.3 \pm 1.2$ & $5.4 \pm 0.6$ & $65.7 \pm 2.1$ & $12.6 \pm 2.6$ & $19.0 \pm 1.4$ & $16.2 \pm 2.2$ \\
\hline SL1313 & Chr4 & $15.4 \pm 2.0$ & $5.4 \pm 1.0$ & $64.7 \pm 1.5$ & $14.7 \pm 3.2$ & $22.0 \pm 1.7$ & $15.3 \pm 1.9$ \\
\hline SL1314 & Chr4 & $16.3 \pm 1.2$ & $5.4 \pm 0.6$ & $69.3 \pm 1.2$ & $16.0 \pm 3.7$ & $22.4 \pm 1.4$ & $14.6 \pm 2.6$ \\
\hline SL1315 & Chr4 & $15.6 \pm 1.2$ & $5.5 \pm 0.6$ & $\underline{70.7 \pm 1.5}$ & $13.3 \pm 0.6$ & $\underline{18.7 \pm 2.6}$ & $13.2 \pm 2.5$ \\
\hline SL1316 & Chr5 & $17.6 \pm 2.0$ & $5.5 \pm 1.0$ & $64.7 \pm 2.3$ & $13.3 \pm 3.7$ & $\underline{25.1 \pm 1.5}$ & $15.2 \pm 1.9$ \\
\hline SL1317 & Chr5 & $14.5 \pm 3.0$ & $\underline{6.3 \pm 1.0}$ & $\underline{71.3 \pm 1.5}$ & $18.7 \pm 3.7$ & $\underline{17.4 \pm 1.1}$ & $12.3 \pm 2.2$ \\
\hline SL1318 & Chr5 & $14.7 \pm 2.3$ & $6.0 \pm 1.1$ & $68.3 \pm 1.5$ & $13.4 \pm 3.4$ & $22.1 \pm 1.0$ & $12.1 \pm 2.0$ \\
\hline SL1319 & Chr6 & $17.2 \pm 3.5$ & $6.0 \pm 1.7$ & $68.3 \pm 2.1$ & $14.0 \pm 2.1$ & $21.8 \pm 3.0$ & $15.1 \pm 2.2$ \\
\hline SL1320 & Chr6 & - & - & - & - & - & - \\
\hline SL1321 & Chr6 & $17.3 \pm 2.0$ & $5.8 \pm 1.0$ & $69.7 \pm 3.1$ & $12.9 \pm 3.2$ & $20.1 \pm 1.3$ & $12.8 \pm 2.4$ \\
\hline SL1322 & Chr6 & $16.6 \pm 4.1$ & $5.6 \pm 2.0$ & $65.7 \pm 1.5$ & $14.5 \pm 2.6$ & $\underline{18.7 \pm 1.4}$ & $\underline{9.4 \pm 2.2}$ \\
\hline SL1323 & Chr7 & $15.5 \pm 3.5$ & $5.5 \pm 1.7$ & $\underline{70.7 \pm 1.5}$ & $12.6 \pm 2.8$ & $19.9 \pm 2.2$ & $\underline{16.2 \pm 3.2}$ \\
\hline SL1324 & Chr7 & $17.6 \pm 3.0$ & $5.4 \pm 1.5$ & - & $13.4 \pm 3.0$ & $22.0 \pm 1.6$ & $18.2 \pm 3.4$ \\
\hline SL1325 & Chr7 & $13.5 \pm 3.5$ & $5.2 \pm 1.7$ & $\underline{76.0 \pm 0.6}$ & $15.9 \pm 2.8$ & $19.0 \pm 1.2$ & $13.9 \pm 2.7$ \\
\hline SL1326 & Chr8 & $16.1 \pm 2.3$ & $5.4 \pm 1.1$ & $68.3 \pm 0.6$ & $15.9 \pm 1.8$ & $20.7 \pm 1.8$ & $15.9 \pm 2.1$ \\
\hline SL1327 & Chr8 & $15.5 \pm 1.2$ & $5.8 \pm 0.6$ & - & $14.1 \pm 2.6$ & $21.0 \pm 1.4$ & $14.0 \pm 2.5$ \\
\hline SL1328 & Chr8 & $15.0 \pm 2.0$ & $5.6 \pm 1.0$ & $62.0 \pm 1.6$ & $13.7 \pm 2.7$ & $17.0 \pm 1.7$ & $12.8 \pm 3.1$ \\
\hline SL1329 & Chr9 & $14.8 \pm 1.2$ & $5.5 \pm 0.6$ & $57.3 \pm 1.2$ & $14.9 \pm 2.8$ & $\underline{25.5 \pm 1.4}$ & $13.3 \pm 1.9$ \\
\hline SL1330 & Chr9 & $14.6 \pm 2.3$ & $\underline{6.1 \pm 1.1}$ & $63.3 \pm 2.3$ & $12.1 \pm 2.8$ & $22.3 \pm 1.5$ & $14.9 \pm 2.3$ \\
\hline SL1331 & Chr9 & $\underline{18.4 \pm 2.0}$ & $6.0 \pm 1.0$ & $58.7 \pm 2.0$ & $\underline{9.8 \pm 1.7}$ & $22.9 \pm 1.5$ & $12.0 \pm 2.0$ \\
\hline SL1332 & Chr10 & $16.4 \pm 3.0$ & $5.5 \pm 1.5$ & $68.0 \pm 2.0$ & $11.5 \pm 2.3$ & $23.3 \pm 1.9$ & $15.3 \pm 2.1$ \\
\hline SL1333 & Chr10 & $15.0 \pm 3.5$ & $5.9 \pm 1.7$ & $\underline{71.0 \pm 1.6}$ & $13.3 \pm 2.2$ & $21.0 \pm 1.3$ & $14.5 \pm 2.8$ \\
\hline SL1334 & Chr10 & $\underline{18.2 \pm 3.0}$ & $5.5 \pm 1.5$ & $\underline{71.0 \pm 2.6}$ & $13.7 \pm 3.3$ & $23.9 \pm 1.7$ & $14.3 \pm 2.8$ \\
\hline SL1335 & Chr11 & $14.8 \pm 2.3$ & $5.5 \pm 1.1$ & $60.0 \pm 1.5$ & $17.8 \pm 2.0$ & $22.3 \pm 1.9$ & $15.3 \pm 3.2$ \\
\hline SL1336 & Chr11 & $\underline{18.3 \pm 2.0}$ & $\underline{6.1 \pm 1.0}$ & $\underline{74.0 \pm 1.6}$ & $\underline{17.4 \pm 1.8}$ & $\underline{18.6 \pm 2.0}$ & $13.9 \pm 2.0$ \\
\hline SL1337 & Chr12 & $18.6 \pm 2.0$ & $5.5 \pm 1.5$ & $66.3 \pm 2.3$ & $14.3 \pm 2.5$ & $21.5 \pm 1.7$ & $12.4 \pm 1.8$ \\
\hline SL1338 & Chr12 & $14.6 \pm 3.5$ & $5.4 \pm 1.7$ & $65.3 \pm 0.6$ & $11.9 \pm 2.3$ & $21.1 \pm 0.9$ & $13.4 \pm 2.2$ \\
\hline SL1339 & Chr12 & $16.5 \pm 2.3$ & $5.8 \pm 1.1$ & $64.7 \pm 0.6$ & $13.6 \pm 2.7$ & $22.5 \pm 1.8$ & $14.5 \pm 2.9$ \\
\hline
\end{tabular}


Table 4 QTLs for eating-quality traits on CSSLs in the 'Takanari' genetic background in 2018

\begin{tabular}{|c|c|c|c|c|c|}
\hline Trait & Locus name & CSSLS & Position & Flanking marker interval & Additive effect \\
\hline \multirow[t]{6}{*}{ Amylose content (\%) } & $9 A C 1-2$ & SL1304 & Chr1 & sd1-RM6321 & 1.1 \\
\hline & $9 A C 3$ & SL1311 & Chr3 & RM2334-RM7389 & 1.7 \\
\hline & qAC9 & SL1331 & Chr9 & RM5657-RM6797 & 1.3 \\
\hline & qAC10 & SL1334 & Chr10 & RM4455-RM6673 & 1.2 \\
\hline & qAC11 & SL1336 & Chr11 & RM1355-RM7443 & 1.3 \\
\hline & $9 A C 12$ & SL1337 & Chr12 & Bb77A02-RM7102 & 1.4 \\
\hline \multirow[t]{3}{*}{ Protein content (\%) } & $q P C 5$ & SL1317 & Chr5 & RM6034-RM3476 & 0.3 \\
\hline & $q P C 9$ & SL1330 & Chr9 & RM3907-RM6235 & 0.2 \\
\hline & $q P C 11$ & SL1336 & Chr11 & RM1355-RM7443 & 0.2 \\
\hline \multirow[t]{9}{*}{ Eating quality score (-) } & $q E Q 1$ & SL1302 & Chr1 & RM1287-RM1297 & -5.0 \\
\hline & $q E Q 2$ & SL1306 & Chr2 & RM5699-RM1379 & -3.0 \\
\hline & qEQ3-1 & SL1308 & Chr3 & RM7332-RM5748 & -3.1 \\
\hline & $q E Q 4$ & SL1315 & Chr4 & RM3839-RM5608 & -3.4 \\
\hline & qEQ5 & SL1317 & Chr5 & RM6034-RM3476 & -3.7 \\
\hline & qEQ7-1 & SL1323 & Chr7 & RM4584-RM6728 & -3.4 \\
\hline & $q E Q 7-2$ & SL1325 & & RM6394-RM7601 & -6.0 \\
\hline & qEQ10 & SL1333, SL1334 & Chr10 & RM5348-RM5620 & -3.5 \\
\hline & qEQ11 & SL1336 & Chr11 & RM1355-RM7443 & -5.0 \\
\hline \multirow[t]{5}{*}{ Stickiness S1 $\left(\mathrm{N} / \mathrm{m}^{2} \times 10^{2}\right)$} & qST2 & SL1306 & Chr2 & RM5699-RM1379 & -1.2 \\
\hline & qST3-1 & SL1308 & Chr3 & RM7332-RM5748 & -1.1 \\
\hline & qST5 & SL1317 & Chr5 & RM6034-RM3476 & -2.2 \\
\hline & qST9 & SL1331 & Chr9 & RM5657-RM6797 & 2.3 \\
\hline & qST11 & SL1335, SL1336 & Chr11 & RM5824-RM6623 & -1.7 \\
\hline \multirow[t]{7}{*}{ Hardness H2 $\left(\mathrm{N} / \mathrm{m}^{2} \times 10^{4}\right)$} & qHA4-1 & SL1312 & Chr4 & RM16260-RM5633 & 1.9 \\
\hline & qHA4-2 & SL1315 & & RM3839-RM5608 & 2.1 \\
\hline & qHA5-1 & SL1316, SL1317 & Chr5 & RM17836-RM18222 & 2.7 \\
\hline & qHA6-2 & SL1322 & Chr6 & RM5957-RM5463 & 2.1 \\
\hline & qHA8 & SL1328 & Chr8 & RM5767-RM4997 & 2.9 \\
\hline & qHA9 & SL1329 & Chr9 & RM23654-RM3907 & -1.4 \\
\hline & qHA11 & SL1336 & Chr11 & RM1355-RM7443 & 2.1 \\
\hline \multirow[t]{6}{*}{ Whiteness (-) } & $q W H 1$ & SL1303 & Chr1 & RM7124-sd1 & 4.2 \\
\hline & $q W H 2-2$ & SL1306 & Chr2 & RM5699-RM1379 & 2.6 \\
\hline & qWH3 & SL1311 & Chr3 & RM2334-RM7389 & 2.8 \\
\hline & qWH4-1 & SL1312 & Chr4 & RM16260-RM5633 & 2.5 \\
\hline & $q W H 6-2$ & SL1322 & Chr6 & RM5957-RM5463 & -0.9 \\
\hline & $q W H 7$ & SL1324, SL1325 & Chr7 & RM5481-RM3826 & 3.5 \\
\hline
\end{tabular}

Positive additive effect means 'Takanari' allele increasing the trait values

chromosome regions as $q W H 1, q S T 2, q H A 4-2$ and qHA11, confirming the genetic effects of these QTLs not only in 'Takanari' but also in several other indica cultivars. All of the CSSLs carrying qWH1, qST2, qHA4-2 and qHA11 except SL2544 showed no significant differences from 'Koshihikari' in amylose contents.

\section{Discussion}

QTLs for Improving Eating Quality in indica Rice Cultivars There is a wide range of phenotypic variations in eating quality traits among rice cultivars, and consumer preferences differ considerably worldwide (Juliano et al. 1993; Calingacion et al. 2014). Eating quality traits are very different even within indica and japonica rice (Calingacion 


\begin{tabular}{|c|c|c|c|c|c|c|c|c|c|c|c|}
\hline \multirow{2}{*}{$\begin{array}{l}\text { Line } \\
\text { No. }\end{array}$} & \multirow{2}{*}{$\begin{array}{l}\text { Donor } \\
\text { cultivar }\end{array}$} & \multicolumn{6}{|c|}{ Eating-quality score } & \multirow{2}{*}{$\begin{array}{c}\text { Amylose } \\
\text { content } \\
(\%)\end{array}$} & \multirow{2}{*}{$\begin{array}{c}\text { Sticki- } \\
\text { ness } \\
\left(\mathrm{N} / \mathrm{m}^{2} \times 10^{2}\right)\end{array}$} & \multirow{2}{*}{$\begin{array}{c}\text { Hard- } \\
\text { ness } \\
\left(\mathrm{N} / \mathrm{m}^{2} \times 10^{4}\right)\end{array}$} & \multirow{2}{*}{$\begin{array}{l}\text { White- } \\
\text { ness } \\
(-)\end{array}$} \\
\hline & & Chr.1 (qWH1) & & 60 & 70 & 80 & 90 & & & & \\
\hline SL3202 & Naba & & $80.7 \pm 3.5$ & & & -1 & & $14.7 \pm 1.9$ & $18.9 \pm 2.7$ & $22.5 \pm 3.2 *$ & * $14.7 \pm 1.5 *$ \\
\hline \multirow[t]{2}{*}{ SL3302 } & Bleiyo & & \multirow[t]{2}{*}{$82.3 \pm 1.5$} & & & 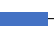 & & $16.6 \pm 1.7$ & $16.7 \pm 3.3$ & $21.7 \pm 2.6 *$ & * $15.7 \pm 1.5 *$ \\
\hline & & Chr.2 (qST2) & & & & & & & & & \\
\hline \multirow[t]{2}{*}{ SL3205 } & Naba & & \multirow[t]{2}{*}{$75.3 \pm 3.2 *$} & & $\mathbf{m}$ & & & $15.1 \pm 0.6$ & $14.1 \pm 2.5 *$ & $19.2 \pm 2.2$ & $13.2 \pm 1.3$ \\
\hline & & Chr.4 (qHA4-2) & & & & & & & & & \\
\hline SL3315 & Bleiyo & & $79.3 \pm 1.5$ & & 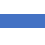 & $-H$ & & $14.0 \pm 1.3$ & $19.5 \pm 3.6$ & $22.6 \pm 3.2 *$ & * $12.3 \pm 1.6$ \\
\hline SL2412 & Bei Khe & & $71.7 \pm 2.1$ & & $=-1$ & & & $14.7 \pm 1.1$ & $20.5 \pm 3.8 *$ & $22.6 \pm 2.9 *$ & * $12.4 \pm 1.5$ \\
\hline \multirow[t]{2}{*}{ SL2520 } & Tupa $121-3$ & & $84.3 \pm 1.2$ & & & & & $15.2 \pm 1.9$ & $21.7 \pm 4.6 *$ & $24.6 \pm 3.2 *$ & * $14.6 \pm 1.1 *$ \\
\hline & & Chr.11 (qHA11) & & & & & & & & & \\
\hline SL2544 & Tupa $121-3$ & & $68.7 \pm 0.6$ & $*$ & +1 & & & $13.0 \pm 1.7$ & * $15.8 \pm 2.1 *$ & $22.8 \pm 2.8 *$ & * $10.8 \pm 0.7$ \\
\hline SL3042 & Basilanon & & $74.3 \pm 0.6$ & & & & & $14.8 \pm 1.3$ & $18.8 \pm 3.2$ & $23.9 \pm 3.3 *$ & $10.5 \pm 1.7$ \\
\hline Koshihikar & & & $85.3 \pm 0.6$ & & 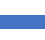 & 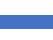 & & $15.0 \pm 2.3$ & $17.0 \pm 3.2$ & $19.3 \pm 2.7$ & $12.4 \pm 1.2$ \\
\hline
\end{tabular}

Fig. 2 Eating quality traits and genotypes of eight CSSLs derived from crosses between the recurrent parent 'Koshihikari' and donor indica rice cultivars. The short arm is on the left and the long arm on the right. White bars, homozygous for the 'Koshihikari' allele; black bars, homozygous for the donor cultivar allele. Data for eating quality traits are presented as means $\pm S D(n=6)$. Red numbers and asterisks indicate significant differences in eating quality traits between CSSLs and 'Koshihikari' at $P<0.05$ by the Dunnet's test

et al. 2014; Hori et al. 2016; Iijima et al. 2019). Generally, cooked grains of japonica cultivars are more sticky and softer than those of indica cultivars (Hori and Yano 2013). In the cultivation areas of japonica cultivars such as China, Korea and Japan, indica cultivars are often evaluated to have lower eating quality than typical japonica cultivars. In this study, we detected six QTLs for eating quality traits involved in differences between indica and japonica rice cultivars using a reciprocal set of CSSLs derived from a cross between a japonica cultivar 'Koshihikari' and an indica cultivar 'Takanari'.

One eating quality QTL, qOE3, has been commonly detected on the short arm of chromosome 3 by using mapping populations derived from crosses between Japanese japonica cultivars (Kobayashi and Tomita 2008; Takeuchi et al. 2008; Wada et al. 2008; Hori and Yano 2013). In these studies, the $q O E 3$ showed the largest genetic effect among the detected QTLs for eating quality and stickiness of cooked rice grains in the previous studies and the 'Koshihikari' allele of $q O E 3$ was associated with good eating quality and strong stickiness of cooked grains. Here, we detected one eating quality QTL, qST3-1, also on the short arm of chromosome 3, and the 'Koshihikari' allele of this QTL was also associated with high eating quality score and strong stickiness of cooked grains. The 'Takanari' allele of $q O E 3$ would be the same as the 'Nipponbare' allele because of consistent haplotypes between these cultivars according to the RAP-DB and TASUKE databases (Sakai et al. 2013; Kawahara et al. 2013; Kumagai et al. 2019). Therefore, qST3-1 is likely identical to qOE3.
We detected five other QTLs with large genetic effects on chromosomes 1, 2, 4, 5 and 11. Both 'Takanari' and 'Koshihikari' segments of the long arm of chromosome 1 containing $q W H 1$ resulted in high whiteness and high eating quality score in the 'Koshihikari' and 'Takanari' genetic backgrounds, respectively. We confirmed the genetic effects of $q W H 1$ in two additional CSSLs carrying chromosome segments derived from indica cultivars 'Naba' and 'Bleiyo'. These data suggest the presence of at least two distinct QTLs for increasing grain whiteness on the long arm of chromosome 1 in japonica and indica rice cultivars. To investigate importance of $q \mathrm{WH1}$ in Japanese rice breeding programs, we investigated genotypes of the $q W H 1$ region in Japanese leading rice cultivars and recently developed rice cultivars (Fig. 3). In the six recently released cultivars, a genome sequence between 37.0 and $39.3 \mathrm{Mbp}$ of the $q W H 1$ region containing the $s d 1$ gene is replaced with indica-type chromosome segments, while the same region in the five leading Japanese cultivars is of japonica type. This difference may be caused not only by selection of semi-dwarf phenotypes caused by the $s d 1$ gene during breeding, but also by selection of grain whiteness caused by $q W H 1$. We cannot be certain whether the QTLs detected in multiple indica cultivars are the same or correspond to different genes. Further genetic analysis, including fine mapping of $q W H 1$, is needed.

The 'Koshihikari' alleles of qST2, qHA4-2, qST5 and qHA11 on the long arms of chromosomes 2, 4, 5 and 11, respectively, improved eating quality traits by increasing stickiness and softening cooked rice grains. There were 

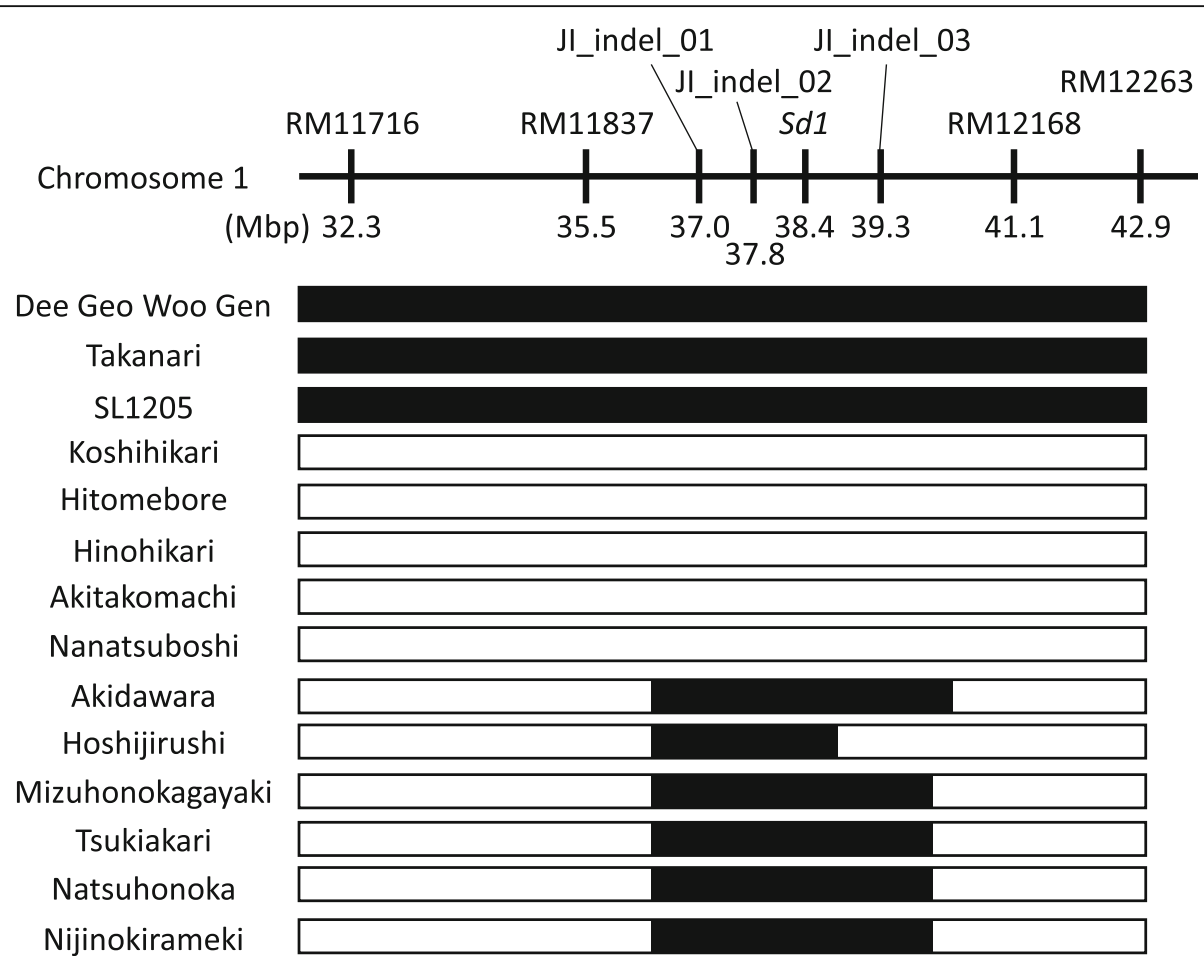

Fig. 3 Genotypes near the sd1 gene on the long arm of chromosome 1 in 'Dee-Geo-Woo-Gen', 'Takanari', SL1205, five Japanese leading cultivars and six high-yielding cultivars released since 2011. The centromere side is on the left, and the distal end of the long arm is on the right. White bars, homozygous for the 'Koshihikari'-type allele; black bars, homozygous for the 'Takanari'-type allele

several reports of QTL detection in mapping populations with the same $W x^{b}$ allele derived from crosses between japonica cultivars. Kwon et al. (2011) found one QTL for eating quality and glossiness of cooked grains on the long arm of chromosome 4. Park et al. (2019) also detected one QTL for eating quality and glossiness of cooked grains on the long arm of chromosome 4, and they fine-mapped another eating quality QTL on the long arm of chromosome 9. Hsu et al. (2014) and Xu et al. (2016) reported QTLs for palatability of cooked grains detected by QTL analysis and genome-wide association study, respectively, in japonica rice cultivars. They reported coexistence of several QTLs and starch biosynthesis genes in the same chromosome regions. Kinoshita et al. (2017) detected three amylose content QTLs and eight protein content QTLs on chromosomes $1-4,6,8,9$ and 12. Takemoto-Kuno et al. (2015) found one amylose content QTL near the centromeric region on the long arm of chromosome 2. These QTLs might not be the same as those detected in our present study, because of difference in chromosome locations.

The QTLs qST2, qST3-1 and qHA4-2 on chromosomes 2, 3 and 4 were not associated with differences in amylose or protein contents. Eating quality traits are quantitative and complex, and are associated with various factors including grain composition and stickiness, hardness and whiteness of cooked grains. In this study, we found QTLs responsible for each of these factors. And, these QTLs might be eating quality genes with different molecular functions from those of previously isolated genes such as $W x, A l k$ and other starch biosynthesis genes, or storage protein genes. Generally, differences in eating quality between japonica and indica rice cultivars seem to be primarily due to different amylose contents caused by allelic differences of the $W x$ and Alk genes. Many studies have reported QTLs for eating quality and starch characteristics corresponding to the $W x$ and Alk genes in mapping populations derived from crosses between indica and japonica rice cultivars (Tan et al. 1999; Wan et al. 2004; Tian et al. 2005; Takeuchi et al. 2007; Su et al. 2011; Yang et al. 2018; Yang et al. 2020). The 'Takanari' allele of the $W x$ gene is identical with the japonica-type $W x^{b}$ allele, and the amylose content is not much different from that of 'Koshihikari' (Aoki et al. 2015; Hori et al. 2016; Iijima et al. 2019). In this study, we did not also detect any QTLs in the chromosome region of the Alk gene, which also greatly affects starch properties. 'Takanari' has A-type in the G / A polymorphism and TT-type in the GC / TT polymorphism of the Alk gene based on genotyping by the DNA marker of Bao et al. (2006) and Hiratsuka et al. (2010). It was different with other weak functional alleles of the japonica-type alk $\left(A l k^{a}\right)$ in 'Koshihikari', Alk $\left(A l k^{c}\right)$ in typical indica type and $A l k^{b}$ in the previous study 
(Chen et al. 2020), but the same weak functional allele as other japonica rice cultivars such as 'Asahi' and 'Akebono' according to the RAP-DB and Rice-TASUKE database (https://ricegenomes.dna.affrc.go.jp/, Sakai et al. 2013; Kawahara et al. 2013; Kumagai et al. 2019). Therefore, 'Koshihikari' and 'Takanari' have the two weak functional alleles of both the $W x$ and Alk genes. This study found novel eating quality QTLs other than the $W x$ and Alk genes. However, we also consider other reasons for no detecting QTLs near the $W x$ and Alk genes. For an example, small genetic effect QTLs in this chromosome region might be concealed by other large genetic effect QTLs. Further studies are required to assess which genes or QTLs are responsible for varietal differences in eating-quality traits.

\section{Common Location of Detected QTLs in indica Rice Cultivars}

The 'Koshihikari' alleles of all QTLs except $q$ WH1 improved eating quality traits. Therefore, the QTLs detected in this study could be used to improve eating quality of many indica cultivars. Because the effect of each single QTL did not improve eating quality to the level of typical japonica cultivars, it would be necessary to accumulate multiple QTL alleles to develop novel indica cultivars with both good eating quality and high grain yield.

Confirmation of $q S T 2, q H A 4-2$ and $q H A 11$ in CSSLs carrying chromosome segments from various indica cultivars suggests common allelic differences in these QTLs between indica and japonica subspecies. The difference in eating quality between japonica and indica cultivars might be due mainly to accumulation of genetic effects of QTLs detected in this study and $W x$ and Alk genes.

\section{Perspectives for Application to Future Rice Breeding}

Although indica cultivars are considered by consumers in Northeast Asian countries such as China, Korea and Japan to have lower eating quality than typical japonica cultivars, many indica cultivars and hybrid rice cultivars have high grain yield (Cheng et al. 2007; Mackill and Khush 2018) and therefore must have genes that increase grain yield. To develop novel rice cultivars that would combine high grain yield and good (japonica-like) eating quality, it is necessary to combine genetic loci that improve eating quality in the background of indica cultivars. Global warming is expected to increase temperatures by $2{ }^{\circ} \mathrm{C}$ by the end of this century (IPCC (Intergovernmental Panel on Climate Change), 2018). Rice cultivars with good eating quality in the indica genetic background would be a good solution to mitigate the effects of climate warming on rice production while preserving the eating quality preferred in Northeast Asia.
However, it is often difficult to select progenies of crosses between indica and japonica rice cultivars. In modern breeding programs, the standard method includes crossing cultivars, fixing genotypes by selfpollination and selecting appropriate lines based on their phenotypes. However, many progenies derived from indica and japonica cultivars have hybrid sterility or hybrid breakdown because of differentiation between the genomes of the two subspecies and incompatibilities in many gene alleles (Matsubara et al. 2007; Yamamoto et al. 2007). If the precise chromosome positions and genetic effects of individual QTLs and genes were revealed, DNA marker technologies could allow us to improve selection efficiency in breeding when using populations derived from crosses between the subspecies. Fine-mapping of individual QTLs will be indispensable in the future. It may also be possible to reproduce favorable alleles by genome editing technology after the responsible genes are identified. Recently, we developed high-speed advanced generation technologies that use a biotron breeding system (Tanaka et al. 2016). These methods would facilitate accumulation of agronomically important genes, such as those for eating quality, grain yield, disease resistance and stress tolerance, across hybridization barriers between subspecies.

\section{Conclusion}

We detected QTLs involved in the control of eating quality traits in CSSLs derived from a cross between a japonica rice cultivar 'Koshihikari' and an indica rice cultivar 'Takanari'. Four of these QTLs, on chromosomes $1,2,4$ and 11, were common in CSSLs derived from several other indica cultivars. These QTLs could be useful for improving eating quality of high-yielding indica cultivars to the level of typical japonica cultivars.

\section{Supplementary Information}

The online version contains supplementary material available at https://doi. org/10.1186/s12284-020-00447-8.

\footnotetext{
Additional file 1: Figure S1. Eating quality score, amylose content, protein content, stickiness of the surface of cooked rice grains, hardness of whole cooked rice grains and whiteness of rice grains in all chromosome segment substitution lines in the 'Koshihikari' genetic background in 2016 (upper) and 2017 (middle), and in the 'Takanari' genetic background in 2018 (lower). Data for eating quality traits are presented as means \pm SD $(n=6)$.

Additional file 2: Table S1. Graphical genotypes of eight CSSLS in the 'Koshihikari' genetic background. Table S2. Eating quality traits and agronomic traits of 41 CSSLs in the 'Koshihikari' genetic background in 2016. Table S3. Eating quality traits and agronomic traits of 41 CSSLs in the 'Koshihikari' genetic background in 2017. Table S4. Eating quality traits and agronomic traits of 39 CSSLs in the 'Takanari' genetic background in 2018. Table S5. Eating quality traits and agronomic traits of eight CSSLs carrying chromosome segments from five indica rice cultivars in the 'Koshihikari' genetic background in 2018.
} 


\section{Abbreviations}

QTL: Quantitative trait locus; CSSL: Chromosome segment substitution line; InDel: Insertion/deletion polymorphism

\section{Acknowledgements}

We are grateful to the technical staff of the Field Management Division at the NARO for their assistance in paddy field work, and to Ms. T Takahashi, Ms. K Shu, Mr. D Nagamatsu, Mr. K Mochizuki and Ms. K Kawamura in the Rice Applied Genomics Research Unit and Rice Quality Research Unit at the National Institute of Crop Science, NARO for their kind assistance in all experiments. We are also grateful to the Hokkaido Research Organization for seed distributions of 'Nanatsuboshi'.

\section{Authors' Contributions}

$\mathrm{KH}, \mathrm{KS}$ and JT designed the experiments. KH, KS, HI, YN, KN and SF developed plant materials, evaluated phenotypes and performed DNA marker experiments. $\mathrm{KH}, \mathrm{KS}$ and JT analyzed the data and wrote the manuscript. The authors read and approved the final manuscript.

\section{Funding}

This study was supported by Grants-in-Aid for Scientific Research (C) (grant numbers 16 K07564 to Kiyosumi Hori and 19 K05984 to Kiyosumi Hori and Junichi Tanaka) from the Japan Society for the Promotion of Science (JSPS).

\section{Availability of Data and Materials}

The all datasets supporting the conclusions of this article are included in the article and supplementary files.

\section{Ethics Approval and Consent to Participate}

Not applicable.

\section{Consent for Publication}

Not applicable.

\section{Competing Interests}

The authors declare that they have no competing interests.

\section{Author details}

${ }^{1}$ Institute of Crop Science, NARO, 2-1-2 Kannondai, Tsukuba, Ibaraki 305-8518, Japan. ${ }^{2}$ College of Agriculture, Ibaraki University, 3-21-1 Chuo, Ami, Ibaraki 300-0393, Japan. ${ }^{3}$ Present address: St. Marianna University School of Medicine, 2-16-1 Sugao, Miyamae, Kawasaki, Kanagawa 216-8511, Japan. ${ }^{4}$ Graduate School of Life and Environmental Science, University of Tsukuba, 2-1-2 Kannondai, Tsukuba, Ibaraki 305-8518, Japan.

\section{Received: 11 May 2020 Accepted: 17 December 2020}

Published online: 07 January 2021

\section{References}

Aoki N, Umemoto T, Okamoto K, Suzuki Y, Tanaka J (2015) Mutants that have shorter amylopectin chains are promising materials for slow-hardening rice bread. J Cereal Sci 61:105-110

Bao J, Corke H, Sun M (2006) Nucleotide diversity in starch synthase lla and validation of single nucleotide polymorphisms in relation to starch gelatinization temperature and other physicochemical properties in rice (Oryza sativa L.). Theor Appl Genet 113:1171-1183

Calingacion M, Laborte A, Nelson A, Resurreccion A, Concepcion JC, Daygon VD, Mumm R, Reinke R, Dipti S, Bassinello PZ et al (2014) Diversity of global rice markets and the science required for consumer-targeted rice breeding. PLoS One 9:e85106

Chen Z, Lu Y, Feng L, Hao W, Li C, Yang Y, Fan X, Li Q, Zhang C, Liu Q (2020) Genetic dissection and functional differentiation of $A L K^{a}$ and $A L K^{b}$, two natural alleles of the $A L K / S S I I^{a}$ gene, responding to low gelatinization temperature in rice. Rice 13:39

Cheng S, Cao L, Zhuang J, Chen S, Zhan X, Fan Y, Zhu D, Min S (2007) Super hybrid rice breeding in China: achievements and prospects. J Integr Plant Biol 49:805-810

Garris AJ, Tai TH, Coburn J, Kresovich S, McCouch S (2005) Genetic structure and diversity in Oryza sativa L. Genetics 169:1631-1638

Goto A, Sasahara H, Shigemune A, Miura K (2009) Hokuriku 193: a new highyielding indica rice cultivar bred in Japan. JARQ 43:13-18
GriSP (Global Rice Science Partnership) (2013) Rice almanac, 4th edn International Rice Research Institute, Philippines

Hiratsuka M, Umemoto T, Aoki N, Katsuta M (2010) Development of SNP markers of starch synthase $/ 1 a(a / k)$ and haplotype distribution in Rice Core collections. Rice Genet Newsl 25:80-82

Hori K, Kataoka T, Miura K, Yamaguchi M, Saka N, Nakahara T, Sunohara Y, Ebana K, Yano M (2012) Variation in heading date conceals quantitative trait loci for other traits of importance in breeding selection of rice. Breed Sci 62:223-234

Hori K, Suzuki K, lijima K, Ebana K (2016) Variation in cooking and eating quality traits in Japanese rice germplasm accessions. Breed Sci 66:309-318

Hori K, Yano M (2013) Genetic improvement of grain quality in japonica rice. In: Varshney RK, Tuberosa R (eds) Translational genomics for crop breeding, Vol. Il abiotic stress, yield and quality. Wiley Blackwell, USA

Hsu YC, Tseng MC, Wu YP, Lin MY, Wei FJ, Hwu KK, Hsing Yl, Lin YR (2014) Genetic factors responsible for eating and cooking qualities of rice grains in a recombinant inbred population of an inter-subspecific cross. Mol Breed 34: 655-673

lijima K, Suzuki K, Hori K, Ebana K, Kimura K, Tsujii Y, Takano K (2019) Endosperm enzyme activity is responsible for texture and eating quality of cooked rice grains in Japanese cultivars. Biosci Biotechnol Biochem 83:502-510

Imbe T, Akama Y, Nakane A, Hata T, Ise K, Ando I, Uchiyamada H, Nakagawa N, Furutachi H, Horisue N et al (2004) Development of a multipurpose highyielding rice variety "Takanari". Bull Natl Inst Crop Sci 5:35-51

IPCC (Intergovernmental Panel on Climate Change) (2018) Global warming of $1.5^{\circ} \mathrm{C}$. https://report.ipcc.ch/sr15/pdf/sr15_spm_final.pdf. Accessed 6th Mar 2020

IRGSP (International Rice Genome Sequencing Project) (2005) The map-based sequence of the rice genome. Nature 436:793-800

Juliano BO, Perez CM, Cuevas-Perez F (1993) Screening for stable high head rice yields in rough rice. Cereal Chem J 70:650-655

Kawahara Y, de la Bastide M, Hamilton JP, Kanamori H, McCombie WR, Ouyang S, Schwartz DC, Tanaka T, Wu J, Zhou S et al (2013) Improvement of the Oryza sativa Nipponbare reference genome using next generation sequence and optical map data. Rice 6:4

Khush GS (1997) Origin, dispersal, cultivation and variation of rice. Plant Mol Biol 35:25-34

Khush GS (1999) Green revolution: preparing for the 21st century. Genome 42: 646-655

Kinoshita N, Kato M, Koyasaki K, Kawashima T, Nishimura T, Hirayama Y, Takamure I, Sato T, Kato K (2017) Identification of quantitative trait loci for rice grain quality and yield-related traits in two closely related Oryza sativa L. subsp. japonica cultivars grown near the northernmost limit for rice paddy cultivation. Breed Sci 67:191-206

Kobayashi A, Hori K, Yamamoto T, Yano M (2018) Koshihikari: a premium shortgrain rice cultivar - its expansion and breeding in Japan. Rice 11:15

Kobayashi A, Tomita K (2008) QTL detection for stickiness of cooked rice using recombinant inbred lines derived from crosses between japonica rice cultivars. Breed Sci 58:419-426

Kojima Y, Ebana K, Fukuoka S, Nagamine T, Kawase M (2005) Development of an RFLP-based rice diversity research set of germplasm. Breed Sci 55:431-440

Konishi S, Izawa T, Lin SY, Ebana K, Fukuta Y, Sasaki T, Yano M (2006) An SNP caused loss of seed shattering during rice domestication. Science 312:13921396

Kumagai M, Nishikawa D, Kawahara Y, Wakimoto H, Itoh R, Tabei N, Tanaka T, Itoh T (2019) TASUKE+: a web-based platform for exploring GWAS results and large-scale resequencing data. DNA Res 26:445-452

Kwon SW, Cho YC, Lee JH, Suh JP, Kim JJ, Kim MK, Choi IS, Hwang HG, Koh HJ, Kim YG (2011) Identification of quantitative trait loci associated with rice eating-quality traits using a population of recombinant inbred lines derived from a cross between two temperate japonica cultivars. Mol Cells 31:437-445

Li C, Zhou A, Sang T (2006) Rice domestication by reducing shattering. Science 311:1936-1939

Mackill DJ, Khush GS (2018) IR64: a high-quality and high-yielding mega variety. Rice 11:18

Matsubara K, Ando T, Mizubayashi T, Ito S, Yano M (2007) Identification and linkage mapping of complementary recessive genes causing hybrid breakdown in an intraspecific rice cross. Theor Appl Genet 115:179-186

Mikami T (2009) Development of evaluation systems for rice taste quality. Jpn J Food Eng 4:191-197

Okadome H (2005) Application of instrument-based multiple texture measurement of cooked milled-rice grains to rice quality evaluation. JARQ $39: 261-268$ 
Park S, Park H, Baek M, Jeong JM, Cho YC, Lee GM, Lee CM, Suh JP, Kim CS, Kim SM (2019) Improving the glossiness of cooked rice, an important component of visual rice grain quality. Rice 12:87

Sakai H, Lee SS, Tanaka T, Numa H, Kim J, Kawahara Y, Wakimoto H, Yang CC, Iwamoto M, Abe T et al (2013) Rice annotation project database (RAP-DB): an integrative and interactive database for rice genomics. Plant Cell Physiol 54:e6

Sato H, Suzuki Y, Sakai M, Imbe T (2002) Molecular characterization of Wx-mq, a novel mutant gene for low-amylose content in endosperm of rice (Oryza sativa L.). Breed Sci 52:131-135

Su Y, Rao Y, Hu S, Yang Y, Gao Z, Zhang G, Liu J, Hu J, Yan M, Dong G et al (2011) Map-based cloning proves qGC-6, a major QTL for gel consistency of japonica/indica cross, responds by Waxy in rice (Oryza sativa L.). Theor Appl Genet 123:859-867

Takai T, Ikka T, Kondo K, Nonoue Y, Ono N, Arai-Sanoh Y, Yoshinaga S, Nakano H, Yano M, Kondo M et al (2014) Genetic mechanisms underlying yield potential in the rice high-yielding cultivar Takanari, based on reciprocal chromosome segment substitution lines. BMC Plant Biol 14:295

Takemoto-Kuno Y, Mitsueda H, Suzuki K, Hirabayashi H, Ideta O, Aoki N, Umemoto T, Ishii T, Ando I, Kato H et al (2015) qAC2, a novel QTL that interacts with $W x$ and controls the low amylose content in rice (Oryza sativa L.). Theor Appl Genet 128:563-573

Takeuchi Y, Hori K, Suzuki K, Nonoue Y, Takemoto-Kuno Y, Maeda H, Sato H, Hirabayashi H, Ohta H, Ishii T et al (2008) Major QTLs for eating quality of an elite Japanese rice cultivar, Koshihikari, on the short arm of chromosome 3. Breed Sci 58:437-445

Takeuchi Y, Nonoue Y, Ebitani T, Suzuki K, Aoki N, Sato H, Ideta O, Hirabayashi H, Hirayama M, Ohta $\mathrm{H}$ et al (2007) QTL detection for eating quality including glossiness, stickiness, taste and hardness of cooked rice. Breed Sci 57:231-242

Tan YF, Li JX, Yu SB, Xing YZ, Xu CG, Zhang Q (1999) The three important traits for cooking and eating quality of rice grains are controlled by a single locus in an elite rice hybrid, Shanyou 63. Theor Appl Genet 99:642-648

Tanaka J, Hayashi T, Iwata H (2016) A practical, rapid generation-advancement system for rice breeding using simplified biotron breeding system. Breed Sci 66:542-551

Tian R, Jiang GH, Shen LH, Wang LQ, He YQ (2005) Mapping quantitative trait loci underlying the cooking and eating quality of rice using a $\mathrm{DH}$ population. Mol Breed 15:117-124

Umemoto T (2018) Gene affecting eating and processing qualities. In: Sasaki T, Ashikari M (eds) Rice genomics, genetics and breeding. Springer Nature, Singapore

Umemoto T, Aoki N, Lin HX, Nakamura Y, Inouchi N, Sato Y, Yano M, Hirabayashi H, Maruyama S (2004) Natural variation in rice starch synthase lla affects enzyme and starch properties. Funct Plant Biol 31:671-684

Umemoto T, Yano M, Satoh H, Shomura A, Nakamura Y (2002) Mapping of a gene responsible for the difference in amylopectin structure between japonica-type and indica-type rice varieties. Theor Appl Genet 104:1-8

Wada T, Ogata T, Tsubone M, Uchimura Y, Matsue Y (2008) Mapping of QTLs for eating quality and physicochemical properties of the japonica rice Koshihikari. Breed Sci 58:427-435

Wan XY, Wan JM, Su CC, Wang CM, Shen WB, Li JM, Wang HL, Jiang L, Liu SJ, Chen LM et al (2004) QTL detection for eating quality of cooked rice in a population of chromosome segment substitution lines. Theor Appl Genet 110:71-79

Wang W, Mauleon R, Hu Z, Chebotarov D, Tai S, Wu Z, Li M, Zheng T, Fuentes RR, Zhang $F$ et al (2018) Genomic variation in 3,010 diverse accessions of Asian cultivated rice. Nature 557:43-49

Xu F, Bao J, He Q, Park YJ (2016) Genome-wide association study of eating and cooking qualities in different subpopulations of rice (Oryza sativa L.). BMC Genomics 17:663

Yamamoto E, Takashi T, Morinaka Y, Lin S, Kitano H, Matsuoka M, Ashikari M (2007) Interaction of two recessive genes, $h b d 2$ and $h b d 3$, induces hybrid breakdown in rice. Theor Appl Genet 115:187-194

Yang B, Xu S, Xu L, You H, Xiang X (2018) Effects of Wx and its interaction with SSIII-2 on rice eating and cooking qualities. Front Plant Sci 9:456

Yang Y, Xu X, Zhang M, Xu O, Feng Y, Yuan X, Yu H, Wang Y, Wei X (2020) Genetic basis dissection for eating and cooking qualities of japonica rice in Northeast China. Agronomy 10:423

Zhang H, Zhu YJ, Fan YY, Huang TX, Zhang JF, Xie HA, Zhuang JY (2019) Identification and verification of quantitative trait loci for eating and cooking quality of rice (Oryza sativa). Plant Breed 138:568-576
Zhou H, Xia D, Zhao D, Li Y, Li P, Wu B, Gao G, Zhang Q, Wang G, Xiao J et al (2020) The origin of $W x^{\text {la }}$ provides new insights into the improvement of grain quality in rice. J Integr Plant Biol. https://doi.org/10.1111/jipb.13011

Zhou L, Sheng W, Wu J, Zhang C, Liu Q, Deng Q (2015) Differential expressions among five Waxy alleles and their effects on the eating and cooking qualities in specialty rice cultivars. J Integr Agric 14:1153-1162

\section{Publisher's Note}

Springer Nature remains neutral with regard to jurisdictional claims in published maps and institutional affiliations.

\section{Submit your manuscript to a SpringerOpen ${ }^{\circ}$ journal and benefit from:}

- Convenient online submission

- Rigorous peer review

- Open access: articles freely available online

- High visibility within the field

- Retaining the copyright to your article

Submit your next manuscript at $\boldsymbol{\nabla}$ springeropen.com 